\title{
A Multisymbol Context-Based Arithmetic Coding Architecture for MPEG-4 Shape Coding
}

\author{
Kun-Bin Lee, Member, IEEE, Jih-Yiing Lin, and Chein-Wei Jen, Member, IEEE
}

\begin{abstract}
MPEG-4 shape coding comprises context-based arithmetic encoding (CAE) as its centerpiece. Since the CAE algorithm has a complicated coding procedure and strong data dependency, it is hard to exploit its pipeline and parallel facilities. Furthermore, to encode multiple symbols within one clock cycle, it needs to overcome the issues of extracting multiple contexts of these symbols, deriving multiple probabilities from these contexts, and performing multiple multiplicative range update operations. This paper presents an efficient pipelined multisymbol CAE architecture for real-time MPEG-4 shape encoding. The proposed design is based on the inherent characteristics of binary alpha blocks as well as the numerical properties of the probabilities indexed by the contexts, and it is capable of encoding either a singe symbol or multiple symbols within each clock cycle. To overcome the aforementioned issues under the consideration of the hardware cost and the critical path delay, only symbols with a particular set of contexts are chosen to be processed simultaneously within the same clock cycle. Theoretical analysis shows that the majority of symbols have contexts belonging to this particular set, and therefore CAE processing can be significantly accelerated. An example VLSI implementation of proposed architecture that encodes two symbols within each clock cycle without sacrificing the clock rate can achieve a speedup of 1.47 in comparison with traditional CAE architectures. This particular two-symbol design can support MPEG-4 Main Profile at levels 3 and 4 under extreme and typical conditions, respectively. When synthesized from Verilog RTL design by using TSMC 0.35- $\mu \mathrm{m}$ 1P4M CMOS technology, the design can run at $90 \mathrm{MHz}$.
\end{abstract}

Index Terms-Context-based arithmetic encoding, MPEG-4, shape coding.

\section{INTRODUCTION}

$\mathbf{M}$ PEG-4's object-based scene description allows the transmission of arbitrarily shaped video objects [1], [2]. The purpose of using shape is to promote better subjective picture quality, higher coding efficiency, as well as more user interaction. These advantages make this standard best suited for the needs of mobile applications or browsing multimedia databases on the Internet. Therefore, shape coding can be widely utilized in various consumer electronics devices, such as video telephony, personal digital assistants, set-top box, and video surveillance.

Manuscript received November 24, 2002; revised July 4, 2003. This paper was recommended by Associate Editor J.-U. Hwang. This work was supported by the National Science Council of the Republic of China under Contract NSC-91-2215-E-009-033.

The authors are with the Department of Electronics Engineering, National Chiao Tung University, Hsinchu, Taiwan 30010, R.O.C. (e-mail: kblee@twins.ee.nctu.edu.tw; jinlin@twins.ee.nctu.edu.tw; cwjen@twins.ee.nctu.edu.tw).

Digital Object Identifier 10.1109/TCSVT.2004.841724

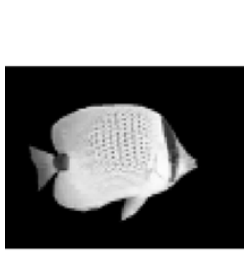

(a)

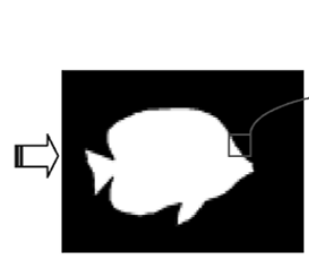

(b)

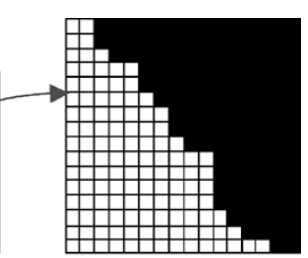

(c)
Fig. 1. Video object plane. (a) Texture. (b) Binary alpha component of the video object. (c) The $16 \times 16 \mathrm{BAB}$.

The MPEG-4 international standard [1], [2] treats moving pictures as an organized collection of visual objects and provides several advanced techniques to access and represent the moving arbitrarily shaped natural and synthetic objects in video scenes. As shown in Fig. 1, a video object plane (VOP), which is the instance of a video object at a given time, is composed of an alpha component to define the object's shape [Fig. 1(b)] and three color components (YCbCr) to render the object's texture [Fig. 1(a)]. MPEG-4 video encoding is based on the VOP encoder shown in Fig. 2. Basically, the VOP is defined as a minimum rectangle that can cover the whole object. The size of a VOP at a given time for a video object depends on the shape of the video object at that time. That is, the size of the VOP for a video object is time-variant. For coding of the shape of a VOP, a bounding rectangle is first created and extended to multiples of $16 \times 16$ blocks with extended alpha samples set to zero. Shape coding is then initiated on a $16 \times 16$ block basis; these blocks are also called binary alpha blocks (BABs), as shown in Fig. 1(c). The alpha component of a VOP is encoded using a binary shape encoder while the color components are encoded using motion estimation and compensation (ME/MC) followed by DCT-based texture coding.

In an MPEG-4 binary shape encoder, a BAB may be encoded in many different ways. If all of the pixels in a BAB are opaque or transparent, only the coding mode is encoded by means of a variable-length coder (VLC). In interframe coding, a $\mathrm{BAB}$ can be coded with reference to a suitable prediction $\mathrm{BAB}$ from the previous coded frame. This procedure is called binary motion estimation (BME). In this case, shape encoder encodes shape mode and motion vector by using VLC. Apart from those cases mentioned above, it is generally necessary to employ context-based arithmetic encoding (CAE) to the pixels within a BAB. There are two CAE operation modes: one is the INTRA mode and the other is the INTER mode. INTRA mode CAE exploits the spatial redundancy by estimating the probability of the current pixel from its neighboring pixels. As for INTER mode, temporal redundancy is exploited by estimating 


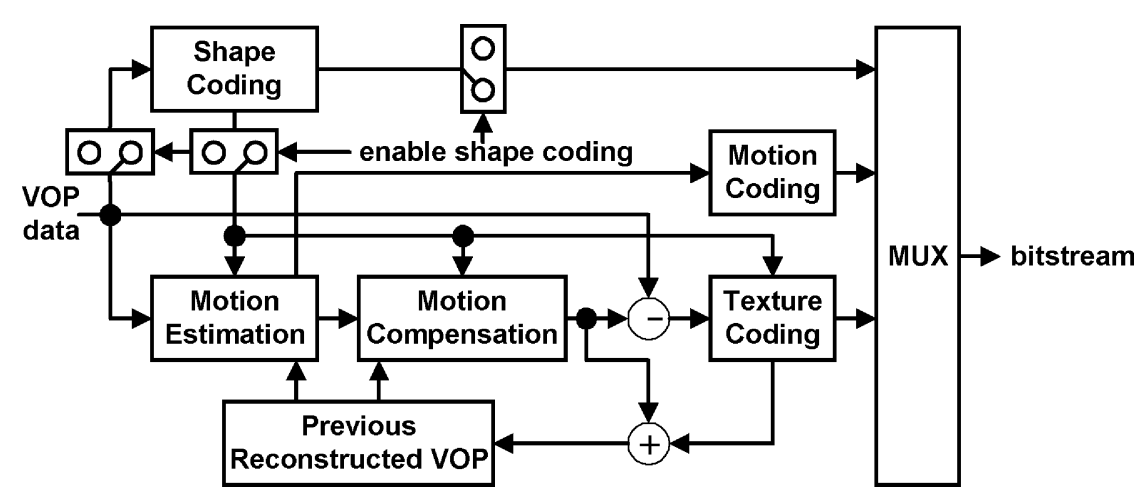

Fig. 2. General structure of an MPEG-4 VOP encoder.

the probability of the current pixel from its neighboring pixels and from motion-compensated neighboring pixels of the previous coded frame.

In intraframe coding, only INTRA mode CAE is performed. In interframe coding, both INTRA and INTER mode CAE are performed. In both modes, CAE operation is performed pixel by pixel in both horizontal and vertical raster scan order. Therefore, there are total four different coding processes: CAE INTRA-H, $\mathrm{CAE}_{\text {INTRA-V }}, \mathrm{CAE}_{\text {INTER-H }}$, and CAE INTER-V. At each pixel, a template is formed. From the template, a context number is extracted and then used to access a probability table (the probability that the pixel is zero). The accessed probability and the value of the pixel are then used to drive an arithmetic encoder. In intraframe coding, the minimum size of the encoded bitstream between CAE INTRA-H $_{\text {and }}$ CAE $_{\text {INTRA-V }}$ is chosen. Similarly, the minimum size of the encoded bitstream among $\mathrm{CAE}_{\text {INTRA-H }}, \mathrm{CAE}_{\text {INTRA-V }}, \mathrm{CAE}_{\text {INTER-H }}$, and CAE INTER-V $_{\text {INT }}$ is chosen in interframe coding. In addition, the shape encoder may decide to encode subsampled versions (i.e., lossy coding) of BABs to save encoded bits. If this is the case, then the subsampling factor is also encoded into the bitstream. The BAB (subsampled or not) then undergoes CAE. Note that CAE itself introduces no additional loss. In summary, the MPEG-4 $\mathrm{CAE}$ algorithm requires a complex processing procedure and high-computation multiplicative operation while suffering severe data dependency.

Similar to MPEG-4's predecessor, i.e., MPEG-2, ME/MC and texture coding are usually implemented by using dedicated hardware accelerators. Dedicated shape coding hardware is also recommended for higher MPEG-4 profiles and levels due to its high computing property [3], [4]. On the one hand, it is shown in [3] that shape encoding for test sequence weather with 10 frames per second (fps) QCIF $(176 \times 144)$ format requires 201.87 MIPS on an Ultra Sparc 2 workstation. On the other hand, [4] showed that CAE ranks the second place in and takes $19.61 \%$ of the total computation complexity of shape encoding in a typical case of MPEG-4 application, i.e., $30 \mathrm{fps}$ CIF $(352 \times 288)$ video sequences. Thus, we can roughly estimate that CAE for test sequence weather with $10 \mathrm{fps}$ QCIF would require 39.59 MIPS on an Ultra Sparc 2 workstation. Therefore, a software CAE implementation for 10-fps QCIF format could be feasible in principle on RISC or DSP platforms. However, the implementation of higher MPEG-4 profiles and levels on general purpose processors might not be feasible. For example,
CAE takes, respectively, 475.08 MIPS and 3263.40 MIPS for $30 \mathrm{fps}$ CIF and $1920 \times 1088$ (main profile at level 4) video sequences, respectively. In addition, the characteristics of bit-level operation and the strong data dependency of CAE also make it hard to be optimized in software implementation. Therefore, to fully utilize the compelling features of MPEG-4 brought about by shape coding, a dedicated hardware design for CAE is imperative.

Several VLSI architectures for the whole MPEG-4 shape coding have also been presented [4]-[6]. For example, the whole shape encoder in [6] is set by a main processor through AMBA AHB bus and synchronized with the processor through interrupt. As for the hardware shape encoder design in [5], CAE takes more cycles (1,144 cycles) than full-search BME ( 1,072 cycles) in interframe coding. Furthermore, BME is not performed in intraframe coding. Therefore, CAE takes a major portion of the execution time in a hardware implementation. The reason why CAE dominates the performance of hardware shape encoder is that, although BME dominates the computing power of the shape encoder, it inherits the good parallelism property from motion estimation, and this property has already been well exploited for hardware design. In contrast, the complicated coding procedure and the strong data dependency of the CAE algorithm make it hard to exploit the pipeline and parallel facilities of CAE hardware design. Since MPEG-4 will be used in a variety of consumer electronics devices, such as video telephony, PDA, and cable boxes, there is a clear need of a cost-effective CAE VLSI architecture to encode shape information in real time.

The aforementioned compelling advantages of MPEG-4 shape coding and the awareness of the critical issues in designing high-performance and lost-cost shape encoder motivate us to explore a cost-effective hardware architecture for CAE. The contribution of this paper is the innovation of a cost-effective, high-speed, multisymbol CAE architecture that can support MPEG-4 main profile at levels 3 and 4 under extreme and typical conditions, respectively. The designs for important implementation issues, including data preparation for contexts of multiple symbols, multiple probabilities lookup for these contexts, and the calculation of multiple multiplications for multiplicative arithmetic coding, are also presented.

The rest of this paper is organized as follows. Section II briefly reviews the related works on the design of arithmetic encoding. Section III presents a general introduction to the 
MPEG-4 context-based arithmetic encoding. Section IV explains the inherent characteristics of $\mathrm{BAB}$ and the numerical properties of the values of probabilities indexed by the contexts. The design space of multisymbol CAE is also explored in this section. Based on the information described in Section IV, a general multiple-symbol CAE architecture is explained first, and the experiment result of a particular two-symbol CAE implementation is shown in Section V. Finally, concluding remarks are given in Section VI.

\section{RELATED WORKS}

To improve the performance of the arithmetic coding, some designs focus on the improvement of clock rate of the multiplication-free arithmetic coding [7]-[9], while other designs focus on parallel-processing multiple symbols within one clock cycle [11]-[14]. Most parallel designs focus on the improvement of the multiplication-free arithmetic coder, the Q-Coder [10]. In the original Q-coder proposed in [10], either a single more probable symbol (MPS) or less probable symbol (LPS) is processed in one iteration. In [11], Feygin modified Q-coder architecture by employing loop unrolling and speculative execution of the inner loop of the arithmetic coding algorithm to achieve either a single LPS or multiple MPSs coding in one iteration. In [12], Jiang incorporated multiplication into the Q-coder coding operation to achieve parallel arithmetic coding. Jiang's architecture has a tree-structure processing element (PE) array, and each PE has its own renormalization operation. Furthermore, Jiang proposed a similar tree-structure parallel design for bilevel images to encode a group of input symbols within each cycle [13]. For input symbols with even number $2 N$, their design requires $4 N$ PEs with a latency of $2+\log 2(2 N)$ cycles. In [14], Andra proposed a modification of the $\mathrm{Q}$-coder by: 1) considering a two-symbol nonoverlapping window and not coding the second symbol if both of them are MPSs and 2) moving the majority of computations to the LPS path. This modification leaded to a $60 \%-70 \%$ reduction of the additions/subtractions with a loss of compression ratio about $1 \%-3 \%$ as compared to the original Q-coder. Andra's design requires a state machine with 58 states while the Q-coder requires a state machine with only 30 states. In addition, the decoder has to make the corresponding changes to correctly extract the coded information.

In MPEG-4, it is necessary to initialize the arithmetic encoder prior to encoding the first pixel of each $\mathrm{BAB}$ and to flush/terminate it after encoding the last pixel of the BAB. Because these frequent initializations and terminations of the arithmetic encoder are a source of inefficiency, a multiplicative arithmetic codec was chosen in preference to the less efficient shift-subtract type [15]. As for MPEG-4 CAE hardware design, Chang [5] proposed the delay line model to improve the context generation of the design in [16], which employed barrel shifters to support the coding of subsampled BABs.

The aforementioned multisymbol designs for multiplicative arithmetic codecs either cause a significant hardware penalty resulting from employing straightforward loop unrolling and speculative execution of the inner loop of arithmetic coding algorithm or result in a loss of compression performance and the corresponding changes being made for the decoder arising from

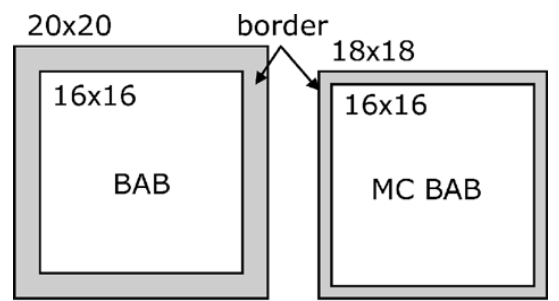

(a)

(b)

Fig. 3. (a) Current bordered BAB. (b) Bordered MC BAB.

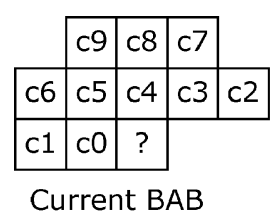

(a)

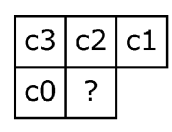

Current BAB
Fig. 4. Templates for defining context. (a) INTRA template. (b) INTER template.

invoking the changes in the algorithm. In addition, all of the above designs do not discuss how to prepare the multiple symbols and contexts for parallel processing. As the number of symbols $N$ grows, it becomes increasingly difficult to get $N$ input symbols, to extract $N$ contexts, to decide whether or not these symbols are suitable for parallel processing, and to process them in parallel with reasonable hardware cost and latency. In this paper, the above-mentioned challenges of hardware design will be efficiently resolved without the sacrifice of compression performance. In addition, the theoretical performance analyses of multisymbol CAE and the effects of renormalization are also revealed.

\section{ConteXt-BASED ARITHMEtic Coding}

This section gives a general introduction to the MPEG-4 context-based arithmetic encoding. The procedure for encoding a given pixel is as follows.

1) A context number is generated based on a template.

2) The probability of the symbol is indexed from a probability table using the context number.

3) The accessed probability and the value of the symbol are used to drive an arithmetic encoder.

To compute the context, the neighboring pixels of the symbol to be coded are used. Therefore, BABs and motion compensation (MC) BABs need to be padded with borders, as shown in Fig. 3, and the padding procedure can refer to the MPEG-4 Video Verification Model [17]. Fig. 4 shows the templates for INTRA and INTER CAE mode. The notation of "?" indicates the symbol to be encoded and is aligned with notation "c6" in INTER template. A 10-b context $C=\sum_{k} c_{k} \cdot 2^{k}$ is generated for INTRA CAE mode while a 9-b context is generated in the same way for INTER CAE mode. The probability table contains the probabilities for a symbol being equal to zero. All probabilities are normalized to the range [1, 65525$]$. Several registers, symbols, and constants are defined in Table I to further describe MPEG-4 CAE algorithm. 

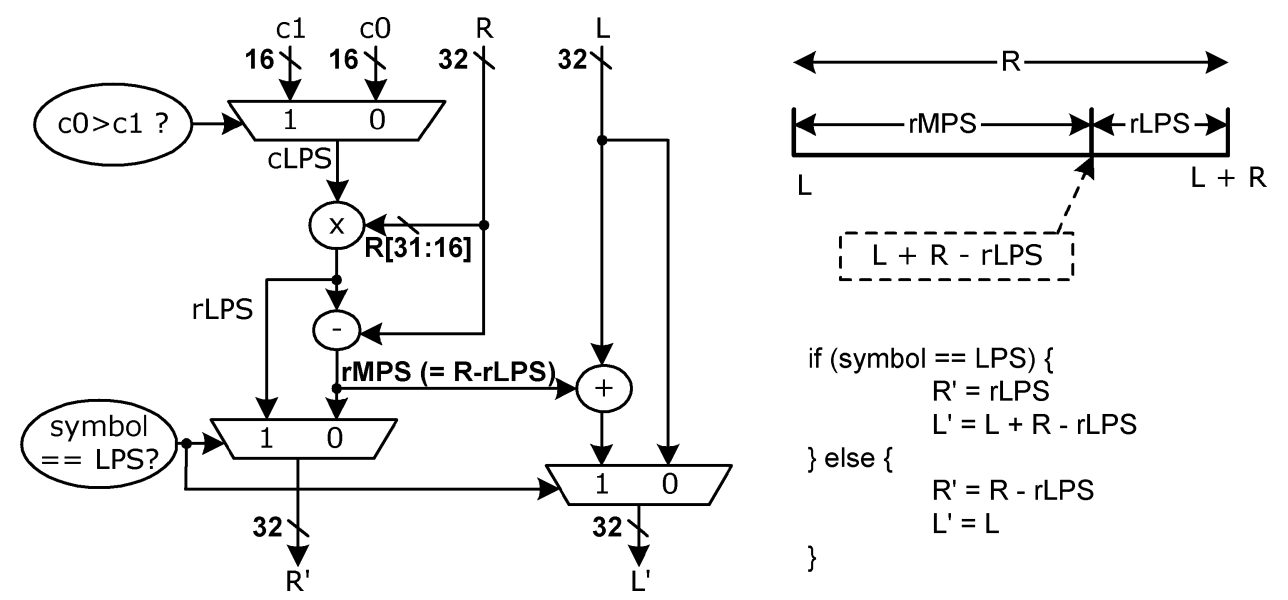

$$
\begin{aligned}
& \text { if (symbol == LPS) \{ } \\
& \mathrm{R}^{\prime}=\mathrm{rLPS} \\
& L^{\prime}=L+R-r L P S \\
& \mathrm{R}^{\prime}=\mathrm{R}-\mathrm{rLPS} \\
& \mathrm{L}^{\prime}=\mathrm{L} \\
& \text { \} }
\end{aligned}
$$

Fig. 5. Range update operation.

TABLE I

VARIABLES FOR THE CAE ALGORITHM

\begin{tabular}{l|l|l}
\hline Notation & Description & Data type \\
\hline HALF & Equal to $1 / 2$ (or $0 \times 80000000)$ & 32-bit fixed point constant \\
\hline QUARTER & Equal to $1 / 4$ (or $0 \times 40000000)$ & 32-bit fixed point constant \\
\hline L & The lower bound of the interval & 32-bit fixed point register \\
\hline $\mathrm{R}$ & The range of the interval. & 32 -bit fixed point register \\
\hline $\mathrm{c} 0$ & Probability for the symbol being equal to 0 & 16-bit fixed point value \\
\hline $\mathrm{cl}$ & Probability for the symbol being equal to 1 & 16 -bit fixed point value \\
\hline LPS & Value of the least probable symbol ('0' or ' 1 ') & 1 -bit Boolean \\
\hline cLPS & Probability of the LPS & 16 -bit fixed point value \\
\hline rLPS & Range corresponding to the LPS & 32-bit fixed point value \\
\hline
\end{tabular}

Once the indexed probability is derived, it is used to drive an arithmetic encoder, which consists of range update and renormalization stages. The range update stage updates the range $(R)$ and the lower bound $(L)$ of the interval. The renormalization stage renormalizes $R$ when $R$ is smaller than QUARTER. This stage could also output the encoded bits of the arithmetic coder. Fig. 5 shows the detailed operations performed in the range update stage. If the input symbol is an LPS, $R$ would be updated to $r L P S$ and $L$ would be updated to a new position with an offset of the range of MPS ( $r M P S$ ) from $L$. If the input symbol is an MPS, $R$ would be updated to $r M P S$ and $L$ would not need to be updated. According to the MPEG-4 standard, $r L P S$ is computed as the product of the MSB $16 \mathrm{~b}$ of $R$ and $c L P S$. Thus, $r M P S$ is calculated as $R$-rLPS.

Fig. 6 shows the operations performed in the renormalization stage that renormalization is handled in three situations. In situation 1 , where the interval $[L, L+R)$ is within [HALF, 1 ), the arithmetic encoder needs to update $L$ and output one bit of 1 along with bits-to-follow. In situation 2, where the interval $[L, L+R)$ is within $[0, \mathrm{HALF})$, the arithmetic encoder needs to output one bit of 0 along with bits-to-follow. Otherwise, renormalization is handled in situation 3 where $L$ is updated and the count of bits-to-follow is increased. After the situation is determined and the corresponding operation is performed, the lower bound and the range need to be enlarged by two times to complete the renormalization operation. Before encoding the next symbol, the renormalization operation is performed iteratively until $R$ is no longer less than QUARTER. The CAE bitstream is generated according to the output bit and
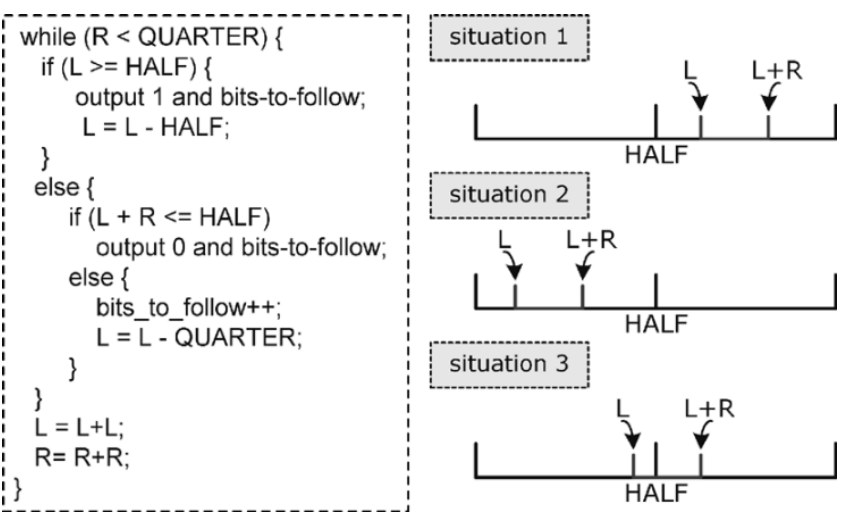

Fig. 6. Renormalization operation.

the value of bits-to-follow, which is the number of the consecutive occurrences of situation 3 . Then the bitstream contains bits-to-follow +1 bits that the value of the first bit is the same as that of the output bit, whereas the value of each remaining bit is opposite to that of the output bit. For example, when the output bit is 1 and the value of bits-to-follow is 4 , then the output bitstream would be a 5-b binary code 10000 .

\section{AnAlysis And DESIGn Space Exploration}

To understand the characteristics of BABs and CAE operations, 11 test sequences listed in Table II are used for the subsequent statistics and analyses. Except for Dancer and Singer, these test sequences are from the MPEG-4 Video Verification Model [17]. All test sequences are generated for the core profile at level two with size conversion disabled and a shape refresh rate of 1:3. In MPEG-4 Video Verification Model, 8-b data are used to represent the alpha component of each pixel to indicate either 0 (opaque) or 255 (transparent). For a hardware design, the bi-level alpha data can be represented as 1-bit 0 or 1 .

\section{A. Multisymbol Context-Based Binary Arithmetic Encoding}

Because the performance bottleneck of CAE is its sequential processing nature, we explore both parallelism and hardware cost to gain an efficient design of multisymbol CAE. As mentioned in Section II, a multiplicative arithmetic encoding was chosen in preference to the less efficient shift-subtract type. 
TABLE II

INFORMATION OF TEST SEQUENCES

\begin{tabular}{lccrrrl}
\hline Sequence name & Abbr. & Frame Size & Frame Number & Total BAB\# & Boundary BAB\# & Description \\
\hline Bream & B & CIF & 10 & 2036 & 569 & A swimming fish \\
Coastguard0 & C0 & CIF & 300 & 77462 & 28150 & River \\
Coastguard1 & C1 & CIF & 300 & 42786 & 14544 & Boat of coastguard \\
Coastguard2 & C2 & CIF & 300 & 15633 & 11781 & Skiff \\
Coastguard3 & C3 & CIF & 300 & 51128 & 8752 & Coast line \\
Dancer 1 & D1 & CIF & 250 & 32885 & 14048 & A performance of two dancers \\
Foreman & F & CIF & 300 & 65958 & 11304 & A man who's talking with body language \\
Singer 1 & S1 & CIF & 250 & 24841 & 10349 & A singer who's singing with body language \\
Singer 2 & S2 & CIF & 250 & 36094 & 10734 & Subtitles that are moving around the singer \\
Stefan & Sf & CIF & 300 & 118800 & 9833 & A man who's playing tennis \\
Stefan_q & Sfq & QCIF & 300 & 5263 & 3477 & QCIF file of Stefan \\
\hline
\end{tabular}

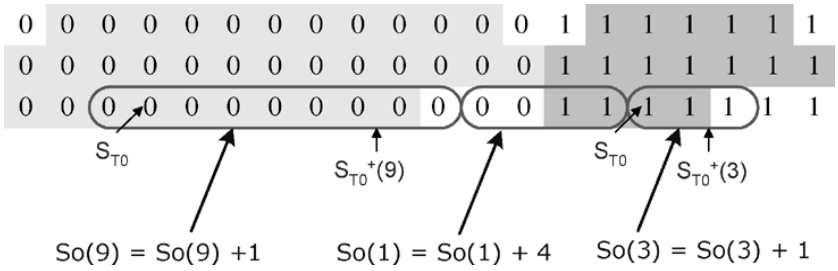

Fig. 7. Examples of counting $S o(n)$ in INTRA mode.

To minimize the hardware cost and the critical path delay of the multiplicative operations to achieve the encoding of multiple symbols within a clock cycle, the design of constant multipliers is preferred. Therefore, only symbols with a particular set of context are chosen to be simultaneously encoded. We denote $S_{T 0}$ as a symbol whose context has either all-zero or all-one bits, $S O(n)$ as the occurrence of $n$ successive $S_{T 0}$ with the same context, and $S c(n)$ as the total symbol count of $S o(n)$, i.e., $S c(n)=S o(n) \times n$. In addition, $S_{T 0}{ }^{+}(n)$ represents a group of $n$ successive $S_{T 0}$ with the same context and can be processed simultaneously within the same clock cycle in our multiple-symbol CAE. Note that $S o(1)$ and $S c(1)$ also record the occurrence and the number of symbols that do not belong to $S_{T 0}$. Fig. 7 illustrates examples for counting $S o(n)$ in INTRA mode. There are nine successive symbols whose contexts have all-zero bits, thus $S o(9)$ is increased by one. In the right-hand side of Fig. 7, there are three successive symbols whose context has all-one bits and therefore $S o(3)$ is increased by one. Because each video object is a rigid body, the neighboring pixels around the coded symbol tend to be all zero or all one, i.e., $S_{T 0}$ should tend to appear in clusters, and this kind of situation would happen to the successive coded symbols. The following statistics also show this point.

Fig. 8 shows the distributions of $S o(n)$ and $S c(n)$ for those test sequences listed in Table II. Although $S o(1)$ dominates the occurrence count, $S c(1)$ has a proportion of $27 \%$ only. On the other hand, the total symbol count of $S_{T 0}{ }^{+}(n)$ has a proportion of $73 \%$; this implies that $S_{T 0}$ tends to appear in clusters. For a rough estimation and comparison of CAE performance without

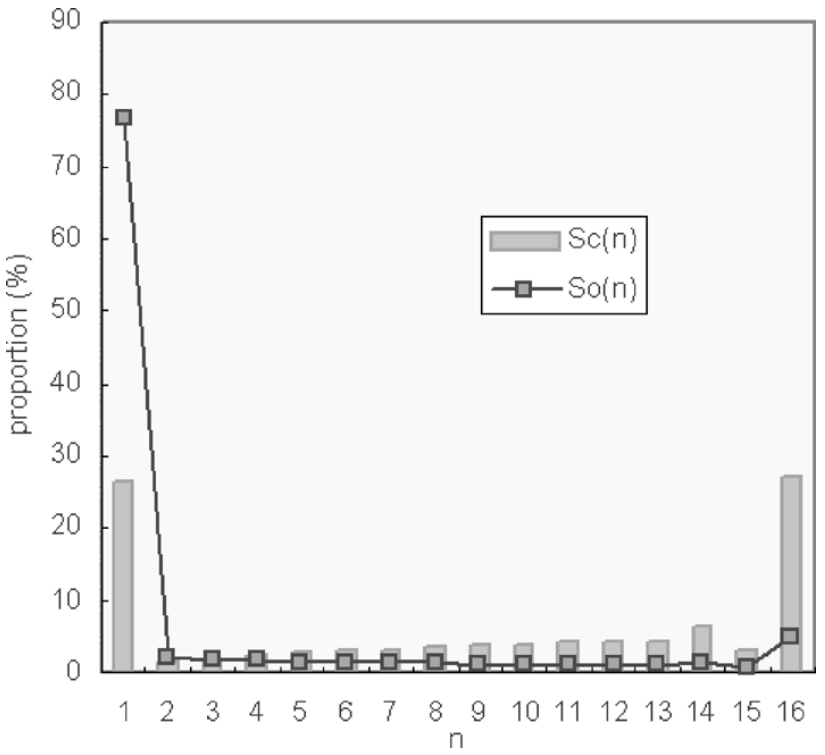

Fig. 8. Distributions of $S c(n)$ and $S o(n)$.

TABLE III CLPS VALUES FOR $S_{T 0}$

\begin{tabular}{c|l|r|r}
\hline \multicolumn{2}{c|}{ Context } & Binary representation & SD representation \\
\hline \multirow{2}{*}{ INTRA } & All 0 & $100,001,101$ & - \\
\cline { 2 - 4 } & All 1 & $11,101,011$ & $100,0 \overline{1} 0, \overline{1} 0 \overline{1}$ \\
\hline \multirow{2}{*}{ INTER } & All 0 & 100 & - \\
\cline { 2 - 4 } & All 1 & 1,110 & $10,0 \overline{1} 0$ \\
\hline
\end{tabular}

considering the renormalization effects and implementation issues, we assume that a baseline CAE can handle one symbol per clock cycle. Then the distribution of $S c(n)$ in Fig. 8 implies that the upper bound performance of a CAE that can handle $S_{T 0}{ }^{+}(2)$ per clock cycle is approximately $27 \% \times 1+73 \% \times 0.5=0.635$ cycles/symbol or 1.575 symbols/cycle, compared with a true two-symbol CAE that has a performance of two symbols/cycle. As for a CAE that can handle $S_{T 0}{ }^{+}(n)$ with arbitrary $n$ within one clock cycle, the speedup is about 2.95 . 


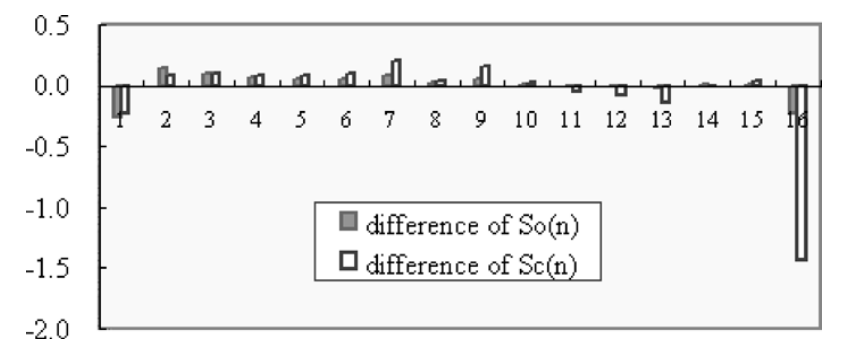

Fig. 9. Differences of $S o(n)$ and $S c(n)$.

Table III shows the cLPS values for $S_{T 0}$ in INTER and INTRA CAE modes. Binary representation of cLPS is replaced by signed-digit (SD) code when the cLPS in SD form has fewer nonzero digits. The mixed representation of cLPS can reduce the cost of the hardwired constant multipliers. Note that the values of cLPSs listed in Table III are quite small compared to those of other cLPSs.

\section{B. Renormalization}

As mentioned in Section III, renormalization operations start when $R$ is smaller than QUARTER and must be completed before the next symbol is encoded. In addition, renormalization operations for one symbol can be completed within either one or more clock cycles according to the implementation. From the statistics of the 11 test sequences, about $6.18 \%$ of coded symbols need to perform renormalizations, and about $0.76 \%$ of coded symbols that need to perform renormalizations belong to $S_{T 0}{ }^{+}(n)$. This implies that the renormalization operations, and thus the renormalization strategies, affect the performance of proposed multiple-symbol CAE design quite little. This is reasonable because the values of cLPSs for $S_{T 0}$ are small and thus arithmetic encoder changes $R$ slightly when encoding $S_{T 0}$ 's. Therefore, the renormalization occurs rarely when encoding $S_{T 0}$ 's. Assuming that one iteration of renormalization operation takes one extra clock cycle to complete the encoding of a symbol and that the total $\mathrm{CAE}$ operation cycles of a $\mathrm{BAB}$ is the summation of the total symbol count and Cycle $_{R N}$, which is the total cycle counts for renormalization, then the proportion of $C y c l e_{R N}$, defined as $C y c l e_{R N} /\left(\right.$ total symbol count + Cycle $\left._{R N}\right)$, is about $10.34 \%$ of the total CAE operation cycles.

The differences of $S o(n)$ and $S c(n)$ shown in Fig. 9 are computed as

$$
\begin{aligned}
\text { difference of } S o(n)= & \text { proportion of } S o_{R N}(n) \\
& - \text { proportion of } S o(n) \\
\text { difference of } S c(n)= & \text { proportion of } S c_{R N}(n) \\
& - \text { proportion of } S c(n)
\end{aligned}
$$

where $S o_{R N}(n)$ and $S c_{R N}(n)$ represent respectively $S o(n)$ and $S c(n)$ with renormalization effect. The negative difference of $S o(1)$ implies that $S o_{R N}(1)$ has less proportion than $S o(1)$. Since renormalizations occurring at $S_{T 0}{ }^{+}(n)$ will break one $S o(n)$ with $n>1$ into several $S o_{R N}(n)$ 's, the total amount of $S o_{R N}(n)$ 's with $n>1$ increases. This results in a reduced proportion of $S o_{R N}(1)$. As illustrated before, renormalizations seldom occur at $S_{T 0}{ }^{+}(n)$, and, even if renormalizations do occur at $S_{T 0}{ }^{+}(n)$, renormalizations break $S_{T 0}{ }^{+}(n)$ with large $n$ into $S_{T 0}{ }^{+}(n)$ 's with smaller $n$ 's most of the time. Therefore, the renormalization operations have very little effect on the performance of proposed multiple-symbol CAE design.

\section{Design Space Exploration for CAE Coding}

To design an efficient multisymbol CAE, three challenges must be conquered:

- extract multiple contexts of symbols and derive the probabilities of these contexts;

- have the critical path in range update operation;

- achieve intersymbol dependency.

If we handle symbols with all kinds of contexts, we would require multiple probability generators, e.g., either multiple probability tables or a multiport probability table, and multiple multipliers. From the fact that $S_{T 0}$ tends to appear in clusters, and this kind of situation would happen to successive symbols, we design a multisymbol CAE that can encode $S_{T 0}{ }^{+}(n)$, a single $S_{T 0}$, or a singe non- $S_{T 0}$ within one clock cycle. The proposed architecture significantly improves the performance and reduces the cost of both multiple probabilities generation and multiplesymbol range update unit.

Fig. 10 shows the proposed pipelined multisymbol CAE architecture. Both bordered current $\mathrm{BAB}$ and bordered $\mathrm{MC} \mathrm{BAB}$ are stored in the Bordered BAB Buffer, and they can be read out one row or one column for horizontal or vertical scan, respectively. Context Generator contains two three-line buffers and can shift the data in the line buffers one or multiple positions to form the INTRA or INTER templates within one clock cycle. After completing the coding of one line of symbols, Context Generator shifts out one line of symbols and reads in another line of symbols from Bordered BAB Buffer. A single context is fed into the probability lookup table (PLT) while multiple contexts are fed into a multiple symbol range update control (MSRUC) unit, to decide whether these symbols belong to $S_{T 0}{ }^{+}(n)$. If there are $n$ symbols belonging to $S_{T 0}{ }^{+}(n)$, Context Generator will shift the data $n$ positions, and these $n$ symbols will be handled in multiple-symbol range update (MSRU) unit. Otherwise, the Context Generator only shifts data one position and only one symbol will be handled in the range update (RU) unit. In both cases, when the updated $R$ is smaller than QUARTER, renormalization operations start in the renormalization (RN) unit. The major differences between the coding paths of $S_{T 0}{ }^{+}(n)$ and non- $S_{T 0}{ }^{+}(n)$, i.e., a single $S_{T 0}$ or a singe non- $S_{T 0}$, are the PLT/RU and MSRUC/MSRU.

As shown in Fig. 5, the critical path of updating the range includes the $16 \times 16$ multiplication, one 32-b subtraction, and one 32-b addition. This is not the case when it happens to encode $S_{T 0}{ }^{+}(n)$. Since the cLPSs for $S_{T 0}$ are small, hardware implementation for these multiplications is compact. Due to the small values of these cLPSs and the fact that $S_{T 0}{ }^{+}(n)$ dominates the total symbol count, it is possible to design a cost-effective MSRU unit that can encode $S_{T 0}{ }^{+}(n)$ within one clock cycle without sacrificing the clock rate.

Before designing a particular MSRU unit, the potential speedup of different capabilities of the $n$-MSRU unit that handles $S_{T 0}{ }^{+}(n)$ within one clock cycle is analyzed. In Fig. 11, 


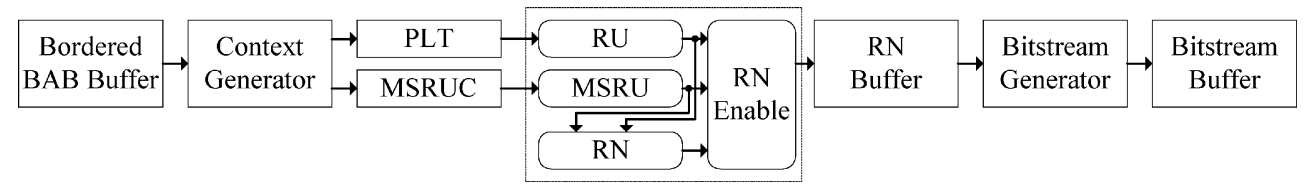

Fig. 10. Proposed multisymbol CAE architecture.

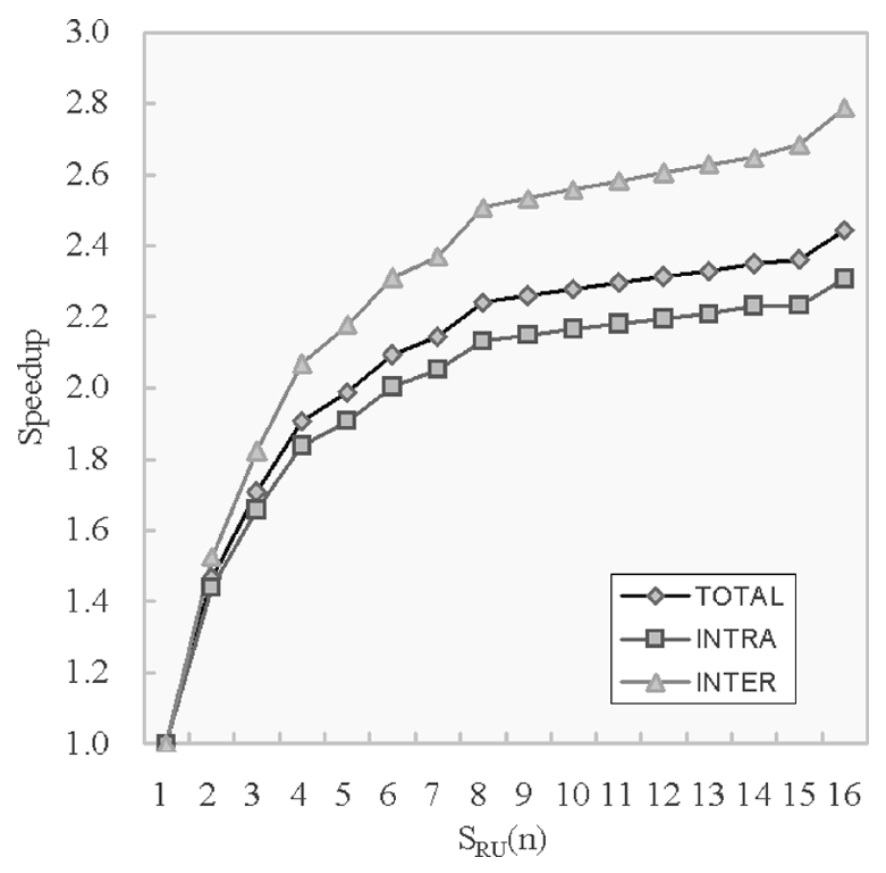

Fig. 11. Speedup of the multisymbol CAE that handles different numbers of symbols.

$S_{R U}(n)$ denotes the number of $S_{T 0}{ }^{+}(n)$ processed by the $n$-MSRU unit in one clock cycle, and Speedup is defined as Cycle(1)/Cycle(n), where Cycle(1) and Cycle(n) represent, respectively, the operation clock cycles of the CAE with the RU unit only and the CAE with both the RU and $n$-MSRU units. Both Cycle(1) and Cycle $(n)$ include cycles for renormalization operations.

Recall that cLPSs for $S_{T 0}$ of INTER CAE are much smaller than those of INTRA CAE. This implies that the $n$-MSRU unit would have a simpler architecture for INTER CAE part. Therefore, it is possible to design an MSRU unit that can handle $S_{T 0}{ }^{+}(m)$ for INTER CAE operation and $S_{T 0}{ }^{+}(n)$ for INTRA CAE operation to achieve a better performance/cost tradeoff. As mentioned in Section I, INTRA CAE operation is applied twice to BABs of intraframe (INTRA BABs) for vertical and horizontal scans; similarly, INTRA CAE and INTER CAE operation are both applied twice to BABs of interframe (INTER $\mathrm{BABs})$. Thus, we can estimate the distribution of CAE operation as follows:

Count of INTRA CAE operation

$$
\begin{aligned}
= & \text { count of INTRA BABs } \times 2 \\
& + \text { count of INTER BABs } \times 2
\end{aligned}
$$

Count of INTER CAE operation

$$
=\text { count of INTER BABs } \times 2 \text {. }
$$

The simulation result of the 11 test sequences shows that the count of INTER CAE operation has a proportion of about $34 \%$. Fig. 11 shows the graph of speedup versus the number of symbols an MSRU unit can handle. The curve of INTER represents the speedup calculated for INTER CAE operation only. Similarly, the curve of INTRA represents the speedup calculated for INTRA CAE operation only. The curve of TOTAL indicates the statistics of combined INTER and INTRA CAE operation. Note that INTER CAE has better speedup than INTRA CAE.

In practical hardware designs, hardware cost and achievable clock rate are important considerations. Since cLPSs of $S_{T 0}$ are much smaller compared to cLPSs of other symbols, under the above considerations, it is feasible to add a range update accelerator that deals with several $S_{T 0}{ }^{+}(n)$ within one clock cycle to the CAE design.

\section{Design of Multisymbol CAE}

In this section, we focus on the detailed design of the major stages of the proposed multisymbol CAE architecture shown in Fig. 10. The major stages of this pipelined architecture are context generator, PLT/MSRUC, RU/RU2, and RN. The detail of this part is shown in Fig. 12. For simplicity, we focus on the explanation of the design of the two-symbol CAE architecture. However, the architecture can be easily extended to general multisymbol CAE architecture for MPEG-4 shape coding. Note that RU2 control and RU2 shown in Fig. 12 correspond to MSRUC and MSRU shown in Fig. 10, respectively.

In Fig. 12, data in both bab buffer and mcbab buffer can be read out row by row or column by column for horizontal or vertical scan, respectively. In the Context Generator stage, line buffers, bab_lines and mcbab_lines, are used to generate INTRA and INTER templates. The counter register is used to indicate the position of these templates and to control how the data in line buffers are shifted. For a two-symbol CAE, this counter register is updated by one or two to indicate that one or two symbols have been processed. The bab_end flag is used by the bitstream generator to indicate the end of a vertical or horizontal scan of a BAB. In the stage of probability table lookup and RU2 control, proper context is selected according to the CAE mode. The selected context is then either fed to $1 \mathrm{~K}$ entries $\times 16 \mathrm{~b}$ probability table for INTRA mode or to a 512 entries $\times 16$ brobability table for INTER mode. To handle $S_{T 0}{ }^{+}(2)$ within a clock cycle, extra pixels context_r $r$ are used to decide whether the two successive symbols, current symbol symbol_c and look-ahead symbol symbol_r $r$, belong to $S_{T 0}{ }^{+}(2)$. Flag $c L P S \_s e l$ is used to indicate that all bits of contexts are all zero or all one. There are three major blocks in the range update stage: $\mathrm{RU}$ is the normal range update unit that handles one symbol at a time, RU2 is the range update unit that handles two symbols at a time, and RN deals with renormalization and bits output. All three blocks will 


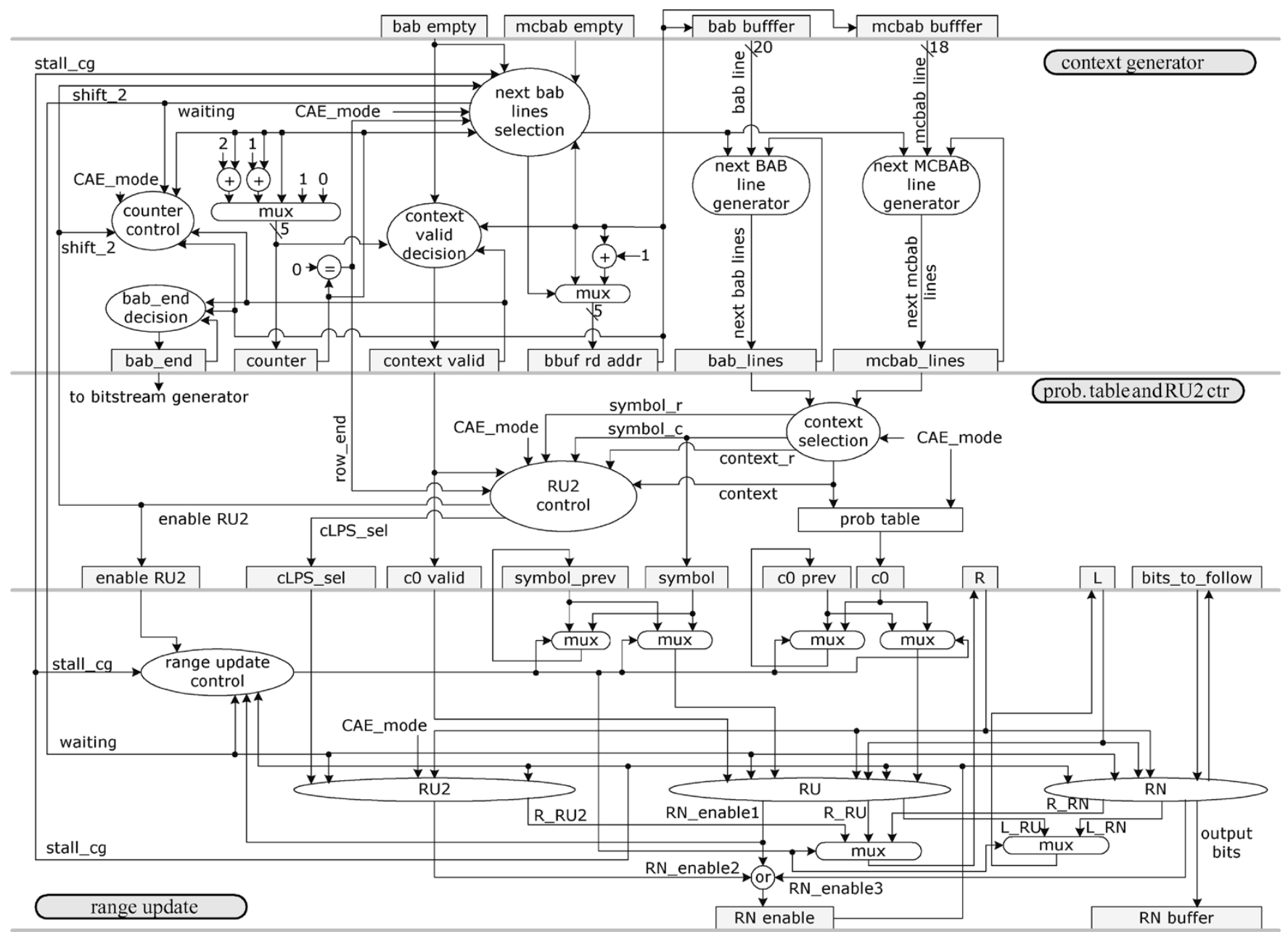

Fig. 12. Major stages of the proposed pipelined multisymbol CAE architecture.

decide the requirement of renormalization after they complete the computation of the range. The whole pipelined stages are stalled when renormalization operation is processing. In the rest of this section, we will discuss the stall conditions and present the detailed design for context generation and range update unit. Although when renormalization occurs, when renormalization buffer is full, or when the BAB line buffers are not ready, the CAE kernel pipeline will be stalled, we will discuss the stalled conditions only caused by renormalization operations.

\section{A. Pipeline Operation}

Fig. 13 illustrates the situation when no renormalization occurs among the input symbols. The symbol pairs $\{\mathrm{S} 1, \mathrm{~S} 2\},\{\mathrm{S} 3$, $\mathrm{S} 4\},\{\mathrm{S} 5, \mathrm{~S} 6\}$ and $\{\mathrm{S} 7, \mathrm{~S} 8\}$ all belong to $S_{T 0}{ }^{+}(2)$. The singlesymbol architecture requires 10 cycles to encode these eight symbols, whereas the two-symbol architecture requires only six cycles. In this situation, the two-symbol architecture can save four cycles since there are four occurrences of two successive symbols belonging to $S_{T 0}{ }^{+}(2)$. Fig. 14 shows the pipeline operations under different situations when the renormalizations are induced by different symbols. In these three situations, only S1 and $\mathrm{S} 2$ belong to $S_{T 0}{ }^{+}(2)$. In case 2, the renormalization is induced by S0. In this situation, both single-symbol and twosymbol architecture take one extra cycle for renormalization.
However, one cycle is saved in the two-symbol architecture due to the occurrence of $\mathrm{S} 1$ and $\mathrm{S} 2$.

In case 3, the renormalization is induced by S1. Therefore, $\mathrm{S} 1$ and $\mathrm{S} 2$ cannot be encoded within a single cycle even though both $\mathrm{S} 1$ and $\mathrm{S} 2$ belong to $\mathrm{S}_{T 0}{ }^{+}(2)$. In such a situation, the two-symbol architecture does not have the advantage over the single-symbol architecture. Note that since the data in the line buffers would be shifted for two symbols when the RU2 control block detects two successive symbols belonging to $S_{T 0}{ }^{+}(2)$, there must be registers to store the symbol and the cLPS of S2 for the second RU operation on S2. In case 4, S2 induces the renormalization. Both single- and two-symbol architecture require one more cycle for the renormalization. However, one cycle is saved in the two-symbol architecture due to the occurrence of $\mathrm{S} 1$ and $\mathrm{S} 2$.

As evidenced by the statistics illustrated in the previous section, renormalization seldom happens to $S_{T 0}{ }^{+}(n)$, and its effect on the performance only causes a little degradation. Together with the pipeline behavior just mentioned, the two-symbol CAE architecture can improve the performance most of the time when encoding two successive symbols that belong to $S_{T 0}{ }^{+}(2)$.

\section{B. Context Generation}

As mentioned before, one of the challenges to design an efficient multisymbol CAE is extracting the multiple contexts of 


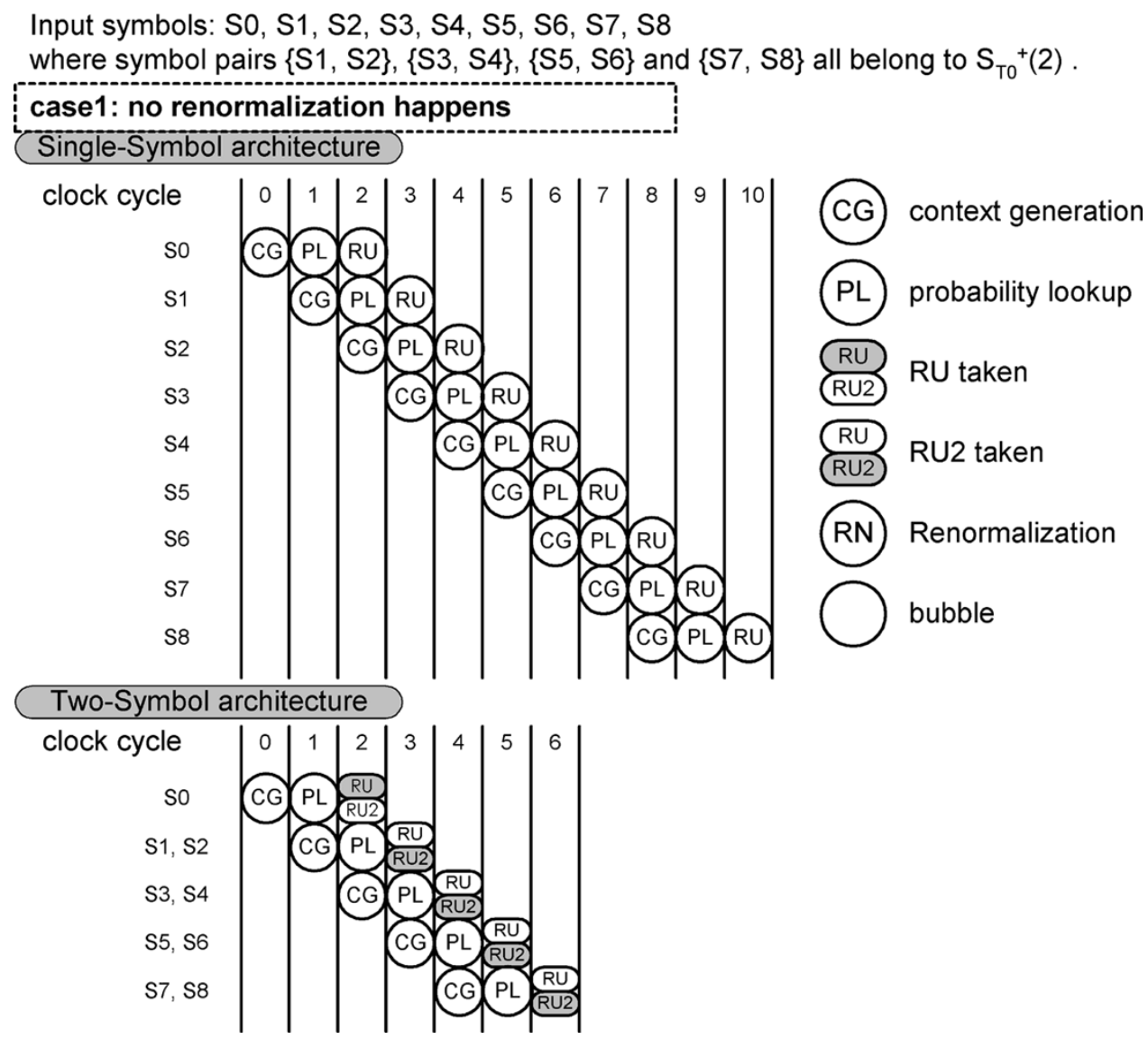

Fig. 13. Pipeline operation without renormalization.

the coded symbols. Fig. 15(a) shows the required pixels to generate two INTRA contexts for symbol $X$ and $X^{\prime}$ within a clock cycle. Virtually, this template will be shifted right by one or two positions respectively through the three line buffers shown in Fig. 15(b) when one or two symbols are encoded or it will be kept at the same position when the CAE is stalled or stopped. These line buffers (LB1, LB2, and LB3) are physically composed of four register chains for the movement of data. The first three register chains, $\mathrm{rc} 1, \mathrm{rc} 2$, and $\mathrm{rc} 3$, are used for border region, and their connection and control are almost independent of the number of symbols to be processed at a time. These three register chains are simple shift registers with control steps shown in Table V. Register $\mathrm{B}_{\mathrm{I} 2}$ is somewhat different because it may receive data from either $\mathrm{B}_{\mathrm{J} 1}$ of $\mathrm{rc} 1$ or $\mathrm{I}_{1}$ of rc4. The rest of registers in these line buffers are mapped as $\mathrm{R}_{1} \sim \mathrm{R}_{16}=\mathrm{I}_{1} \sim$ $\mathrm{I}_{16}, \mathrm{R}_{17} \sim \mathrm{R}_{32}=\mathrm{J}_{1} \sim \mathrm{J}_{16}$, and $\mathrm{R}_{33} \sim \mathrm{R}_{48}=\mathrm{K}_{1} \sim \mathrm{K}_{16}$ to form register chain rc4. Data in rc4 can be shifted either one or two positions. The control steps of generating contexts for symbol $X$ and $X^{\prime}$ are shown in Table IV. At the beginning of processing a $\mathrm{BAB}$, three top rows of the $\mathrm{BAB}$ are loaded into these line buffers in parallel. After the end of encoding one row of the BAB, LB1 is loaded with new data again while data in LB2 and LB3 are already in the right positions. All of these load and shift controls can be easily implemented using a counter. To support $4 \times 4$ and $8 \times 8 \mathrm{BAB}$ sizes, the roles of $\mathrm{J}_{15}, \mathrm{~J}_{16}, \mathrm{I}_{15}$, and $\mathrm{I}_{16}$ are replaced by $\mathrm{J}_{7}, \mathrm{~J}_{8}, \mathrm{I}_{7}, \mathrm{I}_{8}$, and $\mathrm{J}_{3}, \mathrm{~J}_{4}, \mathrm{I}_{3}$, and $\mathrm{I}_{4}$, respectively. Though registers $K_{3} \sim \mathrm{K}_{16}$ do not contribute much to the context generation and can be eliminated, we keep them to
TABLE IV

CONTROL STEP FOR THE INTRA TEMPLATE

\begin{tabular}{l|l|l|l}
\hline & $\left\{\mathrm{C}_{1}, \mathrm{C}_{0}, \mathrm{X}, \mathrm{X}^{\prime}\right\}$ & $\left\{\mathrm{C}_{6}, \mathrm{C}_{5}, \mathrm{C}_{4}, \mathrm{C}_{3}, \mathrm{C}_{2}, \mathrm{C}_{2}\right\}$ & $\left\{\mathrm{C}_{9}, \mathrm{C}_{8}, \mathrm{C}_{7}, \mathrm{C}_{7}\right\}$ \\
\hline Step 1 & $\left\{\mathrm{B}_{\mathrm{K} 1}, \mathrm{~B}_{\mathrm{K} 2}, \mathrm{~K}_{1}, \mathrm{~K}_{2}\right\}$ & $\left\{\mathrm{B}_{\mathrm{J1}}, \mathrm{B}_{\mathrm{J} 2}, \mathrm{~J}_{1}, \mathrm{~J}_{2}, \mathrm{~J}_{3}, \mathrm{~J}_{4}\right\}$ & $\left\{\mathrm{B}_{12}, \mathrm{I}_{1}, \mathrm{I}_{2}, \mathrm{I}_{3}\right\}$ \\
\hline Step 2 & $\left\{\mathrm{B}_{\mathrm{K} 1}, \mathrm{~J}_{16}, \mathrm{~K}_{1}, \mathrm{~K}_{2}\right\}$ & $\left\{\mathrm{B}_{\mathrm{J} 1}, \mathrm{I}_{16}, \mathrm{~J}_{1}, \mathrm{~J}_{2}, \mathrm{~J}_{3}, \mathrm{~J}_{4}\right\}$ & $\left\{\mathrm{B}_{12}, \mathrm{I}_{1}, \mathrm{I}_{2}, \mathrm{I}_{3}\right\}$ \\
\hline Step 3 13 & $\left\{\mathrm{J}_{15}, \mathrm{~J}_{16}, \mathrm{~K}_{1}, \mathrm{~K}_{2}\right\}$ & $\left\{\mathrm{I}_{15}, \mathrm{I}_{16}, \mathrm{~J}_{1}, \mathrm{~J}_{2}, \mathrm{~J}_{3}, \mathrm{~J}_{4}\right\}$ & $\left\{\mathrm{B}_{12}, \mathrm{I}_{1}, \mathrm{I}_{2}, \mathrm{I}_{3}\right\}$ \\
\hline Step 14 & $\left\{\mathrm{J}_{15}, \mathrm{~J}_{16}, \mathrm{~K}_{1}, \mathrm{~K}_{2}\right\}$ & $\left\{\mathrm{I}_{15}, \mathrm{I}_{16}, \mathrm{~J}_{1}, \mathrm{~J}_{2}, \mathrm{~J}_{3}, \mathrm{~B}_{\mathrm{J}}\right\}$ & $\left\{\mathrm{B}_{\mathrm{I}}, \mathrm{I}_{1}, \mathrm{I}_{2}, \mathrm{I}_{3}\right\}$ \\
\hline Step 15 & $\left\{\mathrm{J}_{15}, \mathrm{~J}_{16}, \mathrm{~K}_{1}, \mathrm{~B}_{\mathrm{K} 3}\right\}$ & $\left\{\mathrm{I}_{15}, \mathrm{I}_{16}, \mathrm{~J}_{1}, \mathrm{~J}_{2}, \mathrm{~B}_{\mathrm{J}}, \mathrm{B}_{\mathrm{J} 4}\right\}$ & $\left\{\mathrm{B}_{12}, \mathrm{I}_{1}, \mathrm{I}_{2}, \mathrm{~B}_{13}\right\}$ \\
\hline Step 16 & $\left\{\mathrm{J}_{15}, \mathrm{~J}_{16}, \mathrm{~B}_{\mathrm{K} 3}, \mathrm{~B}_{\mathrm{K} 3}\right\}$ & $\left\{\mathrm{I}_{15}, \mathrm{I}_{16}, \mathrm{~J}_{1}, \mathrm{~B}_{\mathrm{J}}, \mathrm{B}_{\mathrm{J}}, \mathrm{B}_{\mathrm{J}}\right\}$ & $\left\{\mathrm{B}_{\mathrm{I}}, \mathrm{I}_{1}, \mathrm{~B}_{13}, \mathrm{~B}_{13}\right\}$ \\
\hline
\end{tabular}

simplify the transpose design of reading data in either the horizontal or vertical direction from the Bordered BAB Buffer. The context generation of INTER CAE mode is similar to that of INTRA CAE mode.

\section{Range Update Unit}

As shown in Fig. 12, the range update stage includes three major function units: RU, RU2, and RN. RU shown in Fig. 16(a) is implemented in a manner quite like the range update operation introduced in the previous section, except that some stall controls are added. Since RU2 only deals with $S_{T 0}{ }^{+}(2)$, it requires only four different types of constant multiplications with coefficients shown in Table III. These multiplications are individually implemented in intra1 $\mathrm{CU}$, intra0 $\mathrm{CU}$, inter0 $\mathrm{CU}$, and inter $1 \mathrm{CU}$ shown in Fig. 16(b), and the complete functionalities of these CUs are illustrated in Table VI. Since the RU2 deals with two symbols within one clock cycle, there are two CUs for each CU 
Input symbol: S0, S1, S2, S3, where $\mathrm{S} 1$ and $\mathrm{S} 2$ belong to $\mathrm{S}_{\mathrm{TO}^{+}}{ }^{+}(2)$

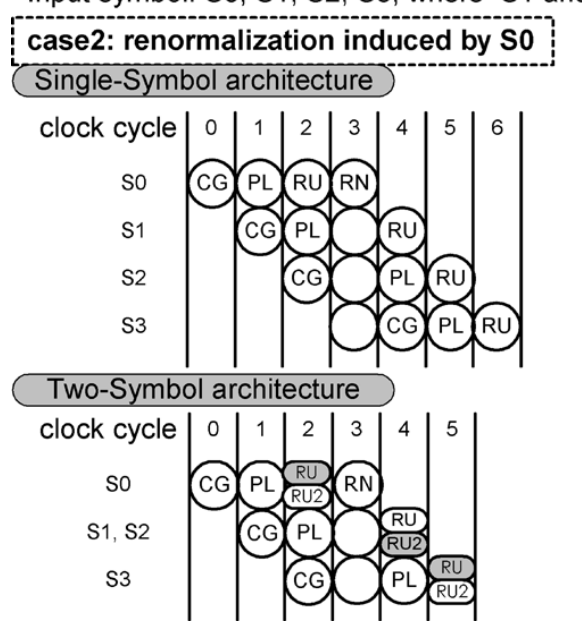

case4: renormalization induced by $\mathrm{S2}$ Single-Symbol architecture

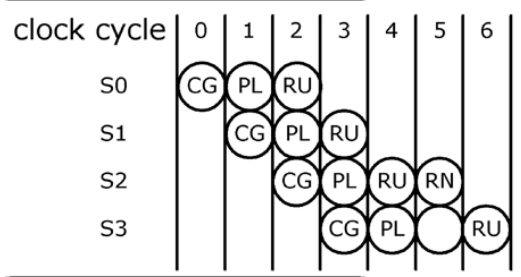

Two-Symbol architecture

clock cycle \begin{tabular}{l|l|l|l|l|l|l|}
0 & 1 & 2 & 3 & 4
\end{tabular}

so

$\mathrm{S} 1, \mathrm{~S} 2$

S3

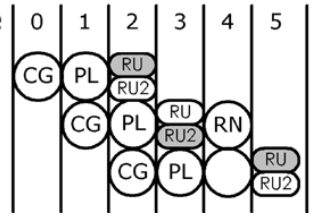

Fig. 14. Pipeline operation with renormalization.

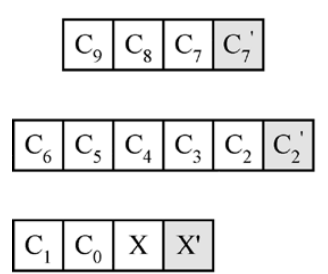

(a)

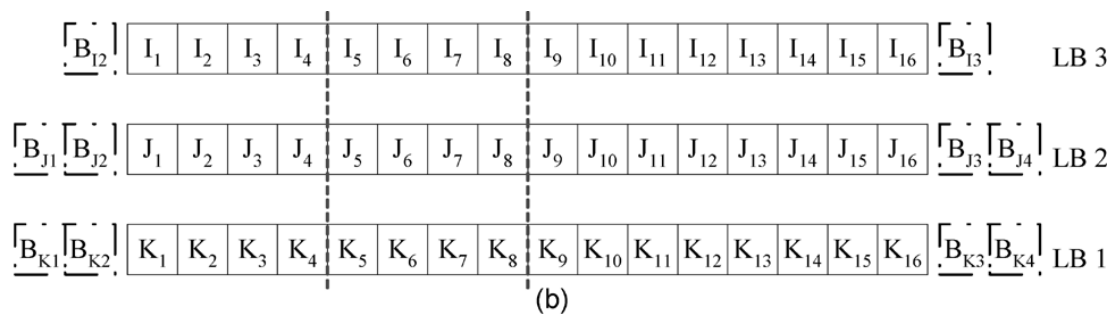

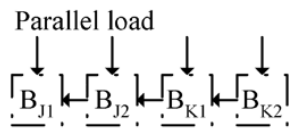

chain $1(\mathrm{rc} 1)$

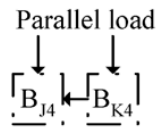

chain $3(\mathrm{rc} 3)$

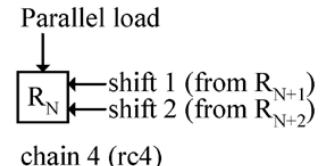

chain 4 (rc4)

(c)

Fig. 15. Context generation for INTRA template.

TABLE $\mathrm{V}$

CONTROL STEP For DATA MOVEMENT OF THE BORDER REgion

\begin{tabular}{l|l|l|l|l|l}
\hline & $\left\{\mathrm{B}_{\mathrm{K} 1}, \mathrm{~B}_{\mathrm{K} 2}\right\}$ & $\left\{\mathrm{B}_{\mathrm{J} 1}, \mathrm{~B}_{\mathrm{J} 2}\right\}$ & $\left\{\mathrm{B}_{\mathrm{J} 3}, \mathrm{~B}_{\mathrm{J} 4}\right\}$ & $\mathrm{B}_{\mathrm{I} 2}$ & $\mathrm{~B}_{\mathrm{I} 3}$ \\
\hline Step 1 & $\left\{\mathrm{B}_{\mathrm{K} 1}, \mathrm{~B}_{\mathrm{K} 2}\right\}_{\text {new }}$ & $\left\{\mathrm{B}_{\mathrm{J} 1}, \mathrm{~B}_{\mathrm{J} 2}\right\}$ new & $\left\{\mathrm{B}_{\mathrm{J}}, \mathrm{B}_{\mathrm{J} 4}\right\}_{\text {new }}$ or $\left\{\mathrm{B}_{\mathrm{K} 3}, \mathrm{~B}_{\mathrm{K} 4}\right\}$ & $\mathrm{B}_{\mathrm{I} 2 \text { new }}$ or $\mathrm{B}_{\mathrm{J} 1}$ & $\mathrm{~B}_{\mathrm{I} 3 \text { new }}$ or $\mathrm{B}_{\mathrm{J} 3}$ \\
\hline Step 2 3 & $\left\{\mathrm{B}_{\mathrm{K} 2}, \mathrm{~B}_{\mathrm{K} 2}\right\}$ & $\left\{\mathrm{B}_{\mathrm{J} 2}, \mathrm{~B}_{\mathrm{K} 1}\right\}$ & $\left\{\mathrm{B}_{\mathrm{J}}, \mathrm{B}_{\mathrm{J} 4}\right\}$ & $\mathrm{I}_{1}$ & $\mathrm{~B}_{\mathrm{J} 3}$ \\
\hline Step 4 16 & $\left\{\mathrm{B}_{\mathrm{K} 2}, \mathrm{~B}_{\mathrm{K} 2}\right\}$ & $\left\{\mathrm{B}_{\mathrm{J} 1}, \mathrm{~B}_{\mathrm{J} 2}\right\}$ & $\left\{\mathrm{B}_{\mathrm{J} 3}, \mathrm{~B}_{\mathrm{J} 4}\right\}$ & $\mathrm{I}_{1}$ & $\mathrm{~B}_{\mathrm{J} 3}$ \\
\hline
\end{tabular}

type. These CUs are implemented in carry-save adder architecture to save area and improve performance. Fig. 17 shows the implementation of RN. RN determines whether to output a one bit of 1 (out1) or 0 (out 0 ) and it counts the bits_to_follow according to the range and lower bound when renormalizing them.

\section{Experiment Result}

The whole CAE design contains Bordered BAB Buffer, Context Generator, probability table and RU2 control block, range update unit, renormalization buffer, bitstream generator, bitstream buffer, and registers for control and status of the whole CAE design. We synthesize the whole CAE design from 20 to $90 \mathrm{MHz}$ with Synopsys using TSMC $0.35-\mu \mathrm{m} 1 \mathrm{P} 4 \mathrm{M}$ CMOS technology, the critical path is dominated by RU, and RU2 takes $9.17 \%-14.26 \%$ of the total area of CAE design. The renormalization buffer has a gate count of 982 for a size of 9 $\mathrm{b} \times 8$ entries, $1 \mathrm{~b}$ for "code bit" and $8 \mathrm{~b}$ for bits_to_follow. In our current design, instead of using ROM and RAM, all of the tables are synthesized using combinational circuitry and all of the buffers are synthesized using registers. The design of these tables and buffers will be refined in the future.

Table VII shows the average clock cycles of CAE operation for different architectures. The average cycle is the time required for one CAE coding process. As mentioned before, two and four $\mathrm{CAE}$ coding processes are required for each $\mathrm{BAB}$ in intraframe and interframe coding, respectively. The bitstream with the minimum size of the encoded bitstream of these coding processes is selected as the final output. The speedup is estimated by dividing the average cycles of each type of architecture by that of proposed architecture with the two-symbol processing feature. The results of the proposed architecture are derived from the Verilog simulation with the test sequence of Bream. Note that the purpose of the proposed architecture with single-symbol 


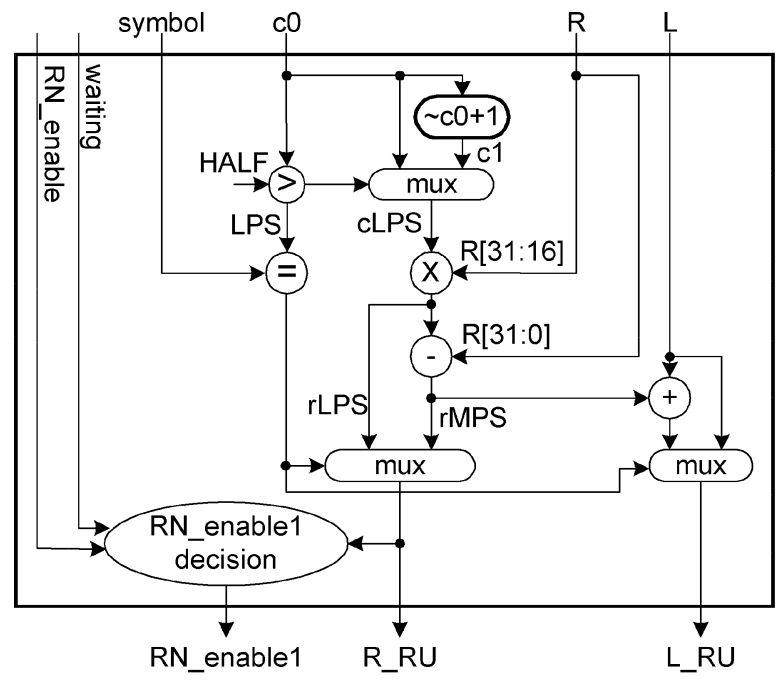

(a)

Fig. 16. (a) RU implementation. (b) RU2 implementation.

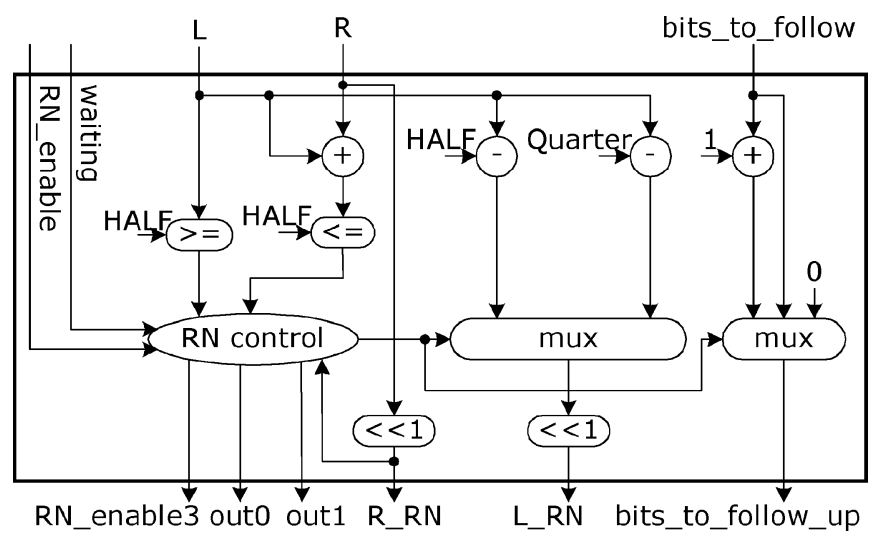

Fig. 17. RN implementation.

processing capability is to demonstrate that the proposed architecture can run at a higher clock rate than the designs in [5], [18], [19], while the purpose of the proposed architecture with two-symbol processing feature is to show that the proposed architecture has higher performance in terms of clock cycle count.

Chou's architecture [18] features its renormalization acceleration by using a lookup table to handle several renormalization operations within one clock cycle. Chang's architecture [19], [5] features its elimination of redundant operations by stopping CAE operation when the size of the current bitstream of the vertical scan is larger than that of the horizontal scan. These features can also be applied to our architecture to achieve further improvement. The idea of redundant operation elimination is to terminate a current coding process when the bitstream size of the current coding process is larger than that of previous coding processes. Applying redundant operation elimination to our two-symbol architecture improves the average clock cycle count for each coding process from 194.65 to 180.33 , i.e., a $7.83 \%$ improvement. The implementation of redundant operation elimination is simple. This mechanism requires one comparator for the bitsream size and the control circuit for terminating the current coding process and enabling the next coding process. The renomalization acceleration proposed

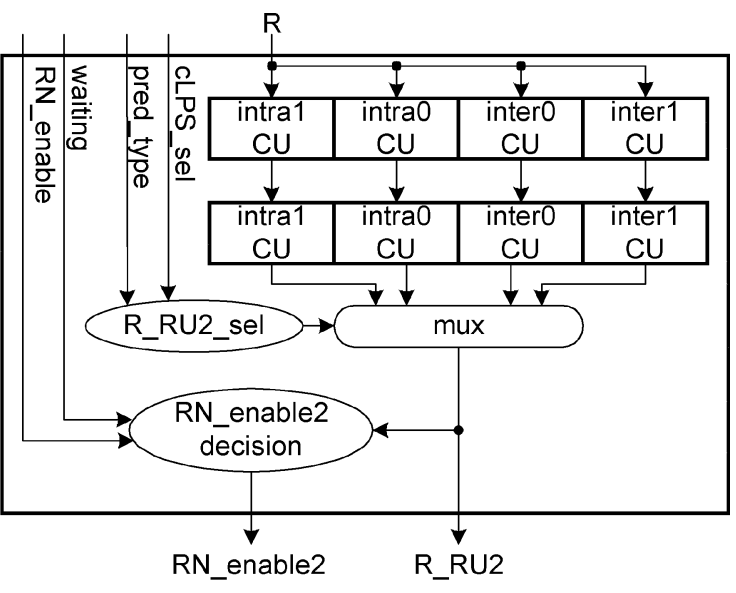

(b)

TABLE VI

FUNCTIONALITIES OF DiFFERENT TYPES OF CU

\begin{tabular}{l|c}
\hline CU Type & Functionality \\
\hline Intra0 & $\mathrm{R}-(\mathrm{R}[31: 16]) \times\left(9^{\prime} \mathrm{b} 1,0000,1101\right)$ \\
\hline Intra1 & $\mathrm{R}-(\mathrm{R}[31: 16]) \times\left(9^{\prime} \mathrm{b} 1,000 \overline{1}, 0 \overline{1} 0 \overline{\mathrm{l}}\right)$ \\
\hline Inter0 & $\mathrm{R}-(\mathrm{R}[31: 16]) \times\left(4^{\prime} \mathrm{b} 0100\right)$ \\
\hline Inter1 & $\mathrm{R}-(\mathrm{R}[31: 16]) \times\left(5^{\prime} \mathrm{b} 1,00 \overline{1} 0\right)$ \\
\hline
\end{tabular}

in [18] is to handle consecutive renormalization processes within one clock cycle. We have illustrated that the ideal case of this improvement is $10.34 \%$ for single-symbol architecture, discussed in Section IV-B. As for Chou's architecture, renomalization acceleration has $4.72 \%$ improvement [18]. The implementation of renomalization acceleration is much more complex than that of redundant operation elimination. Furthermore, the authors of [18] did not clearly illustrate how to implement this improvement.

The gate count listed in Table VII contains the CAE major parts, i.e., context generation, probability lookup, and range update unit. All of these design are synthesized with the same technology library, i.e., TSMC $0.35-\mu \mathrm{m} 1 \mathrm{P} 4 \mathrm{M}$ CMOS. Note that the major parts of the proposed two-symbol architecture require a 12447 gate count when synthesized at $40 \mathrm{MHz}$. The maximum clock rate of Chou's, Wang's, and our design are 72.5, 40, and $90 \mathrm{MHz}$, respectively. The layout and specification of the prototyping chip for the whole CAE design are shown in Fig. 18 and Table VIII, respectively.

\section{CONCLUSION}

In this paper, we have presented an efficient architecture design of multisymbol context-based arithmetic coding for MPEG-4 shape encoding. The important implementation issues, including data preparation for contexts of multiple symbols, multiple probabilities lookup for these contexts, and the calculation of multiple multiplications for multiplicative arithmetic coding, were all efficiently resolved in the proposed 
TABLE VII

COMPARISONS AMONG DiFFERENT CAE ARCHITECTURES

\begin{tabular}{l|c|c|c|r|l}
\hline \hline \multicolumn{1}{c|}{ Architecture } & $\begin{array}{c}\text { Average } \\
\text { cycles }\end{array}$ & Speedup & Gate count & \multicolumn{1}{c|}{$\begin{array}{c}\text { Clock rate } \\
(\mathrm{MHz})\end{array}$} & Features \\
\hline \hline Chou [18] & 307.70 & 1.58 & 17,065 & 72.5 & Renormalization acceleration \\
\hline Chang [19][5] & 286.00 & 1.47 & 11,436 & 40 & Redundant operations elimination \\
\hline \multirow{2}{*}{$\begin{array}{l}\text { Proposed } \\
\text { architecture }\end{array}$} & 285.51 & 1.47 & $17,123 / 12,447$ & $90 / 40$ & Single-symbol processing \\
\cline { 2 - 6 } & 194.65 & 1 & $17,123 / 12,447$ & $90 / 40$ & Two-symbol processing \\
\hline
\end{tabular}

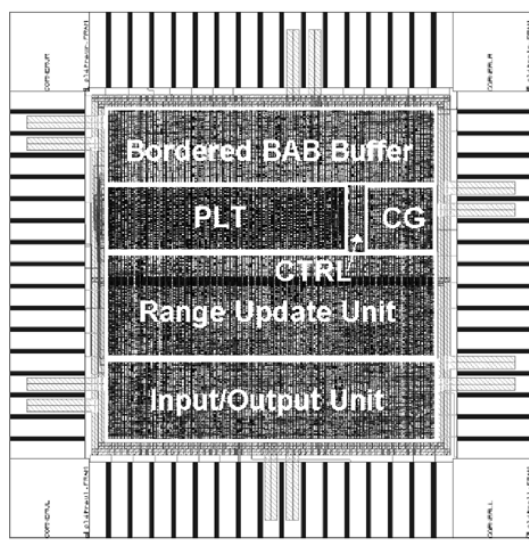

Bordered BAB buffer: Current and MC BAB buffer

PLT: $\quad$ Probability lookup table

CG:

Context generator

CTRL:

Central and RU2 control

Range Update Unit: $\quad$ RU, RU2, and RN

Input/Output Unit: Renormalization buffer

Bitstream generator

Bitstream buffer

Registers for control \& status

Fig. 18. Specification of the prototyping chip.

TABLE VIII

SPECIFICATIONS OF THE PROTOTYPING CHIP

\begin{tabular}{ll}
\hline Technology: & TSMC $0.35 \mu \mathrm{m}$ 1P4M CMOS \\
Gate Count: & 37,617 \\
Die area: & $2.58 \times 2.58 \mathrm{~mm}^{2}$ \\
Supply voltage: & $3.3 \mathrm{~V}$ \\
Maximum clock rate: & $90 \mathrm{MHz}$ \\
Power consumption & $70.15 \mathrm{~mW} @, 90 \mathrm{MHz}$ \\
& \\
Probability Tables & $1 \mathrm{~K} \times 16$ bits \\
$\quad$ INTRA mode & $512 \times 16$ bits \\
INTER mode & \\
Buffers & $20 \times 20$ bits \\
$\quad$ Boarded current BAB buffer & $18 \times 18$ bits \\
Boarded MC BAB buffer & $8 \times 9$ bits \\
Renormalization buffer & $2 \times 298$ bits \\
Bitstream buffer &
\end{tabular}

design. The key idea of our design is to exploit the inherent characteristics of BABs and the numerical properties of the probabilities indexed by the contexts. Data preparation of contexts for multiple symbols is efficiently solved by using four register chains. The design decision that CAE only encodes multiple symbols with the same context settles the issue of the generation of multiple probabilities of multiple symbols at a time and significantly reduces the cost of the probability table. Furthermore, the choice to encode multiple symbols with particular contexts greatly reduces the hardware cost and the required computation time for performing multiple multiplication operations within each clock cycle.

An example VLSI implementation of the proposed architecture that encodes two symbols within each clock cycle without sacrificing the clock rate can achieve a speedup of 1.47 in comparison with traditional CAE architectures. Under the extreme condition of encoding each boundary BAB in four coding processes (CPs), this two-symbol architecture can support Main Profile at Level 3 when running at $37.91 \mathrm{MHz}(48600 \mathrm{MB} / \mathrm{s}$ $\times 4 \mathrm{CP} / \mathrm{MB} \times 195$ cycles $/ \mathrm{CP} \approx 37.91 \times 10^{6}$ cycles/s). To support Main Profile at Level 4 under similar conditions, the design has to be able to run at $190.94 \mathrm{MHz}$. On the other hand, the simulation statistics over the 11 test sequences listed in Table II show that $13.9 \%$ and $10.2 \%$ BABs require two and four coding processes, respectively. To support MPEG-4 Main Profile at Level 4 shape encoding based on this criterion, the proposed two-symbol design has to be able to run at least $65.49 \mathrm{MHz}$ $(489600 \mathrm{MB} / \mathrm{s} \times(2 \times 13.9 \%+4 \times 10.2 \%) \mathrm{CP} / \mathrm{MB} \times 195$ cycles/CP $\approx 65.49 \times 10^{6}$ cycles/s) for real-time MPEG-4 encoding. The exact frequency at which the proposed design has to be able to run depends on the system overhead, such as data transfer of BABs and synchronization among functional units for the overall MPEG-4 encoding and other system functionality.

The major parts of our design requires a 12447 gate count when synthesized at $40 \mathrm{MHz}$, which is about $108.84 \%$ of the design proposed in [5], [19] and 72.94\% of the design proposed in [18]. That is, the proposed two-symbol architecture has small area overhead. Note that the proposed architecture also has a higher maximum clock rate. The aforementioned hardware cost does not include the buffers. As shown in Fig. 9, the input buffer of CAE, called the BAB buffer, requires a 10626 gate count, while the output buffer, called the bitstream buffer, requires a 6122 gate count. Unlike the major parts of CAE, the area of these buffers is less sensitive to the operating clock frequency. That is, the buffers could dominate the hardware cost of CAE. To have two simpler CAE encoders with single-symbol architecture, the size of these buffers would be probably two times of that of the proposed two-symbol architecture. In addition, two 
simpler single-symbol CAE encoders also require almost two times the area for the major parts of CAE. Therefore, the proposed design is a cost-effective solution for MPEG-4 Main Profile at Levels 3 and 4.

\section{REFERENCES}

[1] "Information Technology - Coding of Audio-Visual Objects,", Switzerland, ISO/IEC 14 496-2, 2nd ed., 2001.

[2] N. Brady, "MPEG-4 standardized methods for the compression of arbitrarily shaped video objects," IEEE Trans. Circuits Syst. Video Technol., vol. 9, no. 6, pp. 1170-1189, Dec. 1999.

[3] P. M. Kuhn and W. Stechele, "Complexity analysis of the emerging MPEG-4 standard as a basis for VLSI implementation," in Proc. SPIE Vis. Commun. Image Process. (VCIP'98), Jan 1998, pp. 498-509.

[4] D. Gong and Y. He, "Computation complexity analysis and VLSI architectures of shape coding for MPEG-4," in Proc. SPIE VCIP'2000, vol. 4067, Jun. 2000, pp. 1459-1470.

[5] H.-C. Chang, Y.-C. Chang, Y.-C. Wang, W.-M. Chao, and L.-G. Chen, "VLSI architecture design of MPEG-4 shape coding," IEEE Trans. Circuits Syst. Video Technol., vol. 12, no. 9, pp. 741-751, Sep. 2002.

[6] K.-B. Lee, N. Y.-C. Chang, H.-Y. Chin, H.-C. Hsu, and C.-W. Jen, "Optimal frame memory and data transfer scheme for MPEG-4 shape coding," in Proc. IEEE Int. Conf. Consumer Electronics (ICCE), Jun. 17-19, 2003, pp. 164-165.

[7] M. Tarui, M. Oshita, T. Onoye, and I. Shirakawa, "High-speed implementation of JBLG arithmetic coder," in Proc. IEEE TENCON, vol. 2, 1999, pp. 1291-1294.

[8] Y.-T. Hsiao, H.-D. Lin, K.-B. Lee, and C.-W. Jen, "High-speed memorysaving architecture for the embedded block coding in JPEG2000," in Proc. Int. Symp. Circuits Syst. (ISCAS'02), vol. 5, Phoenix, AZ, May 2002, pp. 133-136.

[9] K.-K. Ong, W.-H. Chang, Y.-C. Tseng, Y.-S. Lee, and C.-Y. Lee, "A high throughput low cost context-based adaptive arithmetic codec for multiple standards," in Proc. Int. Conf. Image Process., vol. 1, 2002, pp. 872-875.

[10] J. L. Mitchell and W. B. Pennebaker, "Optimal hardware and software arithmetic coding procedures for the Q-coder," IBM J. Res. Dev., vol. 32, no. 6, pp. 727-736, Nov. 1988.

[11] G. Feygin, P. G. Gulak, and P. Chow, "Architectural advances in the VLSI implementation of arithmetic coding for binary image compression," in Proc. Data Compression Conf. (DCC '94), 1994, pp. 254-263.

[12] J. Jiang and S. Jones, "Parallel design of arithmetic coding," Proc. Inst. Elec.t Eng., pt. E, vol. 141, pp. 327-333, Nov. 1994.

[13] J. Jiang, "Parallel design of Q-coders for bilevel image compression," in Proc. Int. Conf. Parallel Distributed Syst., 1994, pp. 230-235.

[14] K. Andra, T. Acharya, and C. Chakrabarti, "A multi-bit binary arithmetic coding technique," in Proc. Int. Conf. Image Process., vol. 1, Vancouver, BC, Canada, Sep. 2000, pp. 928-931.

[15] N. Brady, F. Bossen, and N. Murphy, "Context-based arithmetic encoding of 2D shape sequences," in Proc. Int. Conf. Image Process., vol. I, Santa Barbara, CA, Oct. 1997, pp. 29-32.

[16] D. Gong and Y. He, "An efficient architecture for real-time content-based arithmetic coding," in Proc. Int. Symp. Circuits Syst. (ISCAS 2000), vol. 3, Geneva, Switzerland, 2000, pp. 515-518.

[17] "MPEG-4 Video Verification Model Version 18.0,", ISO/IEC JTC1/SC29/WG11 N3908, 2001.

[18] H.-L. Chou, "The design and implementation of MPEG-4 shape encoding core," M.S. thesis, National Chiao Tung Univ., Dept. Electron. Eng., Hsinchu, Taiwan, 2001.

[19] Y.-C. Wang, "Architecture design and implementation of MPEG-4 shape coding,” M.S. thesis, National Taiwan Univ., Dept. Elect. Eng., Taipei, Taiwan, 2001.

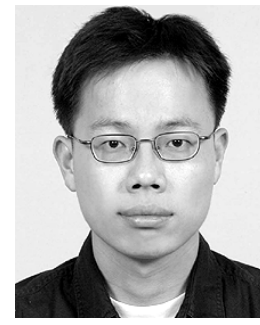

cations.

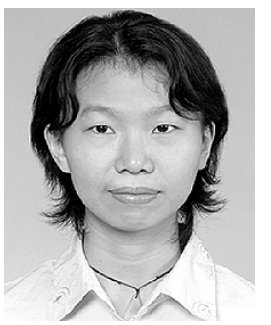

Jih-Yiing Lin received the B.S. degree in electrical engineering and the M.S. degree in electronics engineering from National Chiao-Tung University, Hsinchu, Taiwan, in 2000 and 2002, respectively.

She is currently with Sunplus Technology, Hsinchu. Her research interests include video signal processing, VLSI design, computer architecture, and system-level design methodology.

Ms. Lin is a member of Phi Tau Phi.

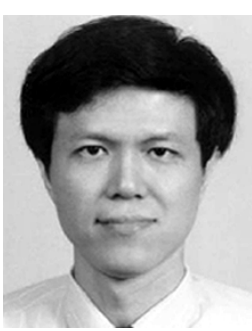

Chein-Wei Jen (S'78-M'84) received the B.S. degree from National Chiao-Tung University, Hsinchu, Taiwan, in 1970, the M.S. degree from Stanford University, Stanford, CA, in 1977, and the Ph.D. degree from National Chaio-Tung University in 1983

$\mathrm{He}$ is currently with the Department of Electronics Engineering and Institute of Electronics, National Chiao Tung University, Hsinchu, Taiwan, as a Professor. During 1985-1986, he was with the University of Southern California, Los Angeles, as a Visiting Researcher. His current research interests include VLSI design, digital signal processing, processor architecture, and design automation.

Dr. Jen is a member of Phi Tau Phi. 